\title{
Impact of diffusion layers in strong electrolytes on the transient current
}

\author{
Matthias Marescaux, ${ }^{*}$ Filip Beunis, Filip Strubbe, Bart Verboven, and Kristiaan Neyts \\ Electronics and Information Systems, Ghent University, Sint-Pietersnieuwstraat 41, Ghent B-9000, Belgium
}

(Received 23 September 2008; published 21 January 2009)

\begin{abstract}
Transient currents of electrolytes in response to a voltage step can reveal a lot about the behavior of charges present in an electrolyte. In this paper, electrolytes with high ionic strength are considered. In the limit of small voltage steps, the interpretation is straightforward as the equations describing the transient can be linearized. However, when high ion concentrations and voltage steps of the order of $k T / q$ are considered, we find higher-order effects that occur simultaneously with the diffuse double layer charging. In this case, the diffuse double layer and the transient diffusion layer are coupled because of the screening of the field, leading to a $-3 / 2$ power law for the transient current.
\end{abstract}

DOI: 10.1103/PhysRevE.79.011502

PACS number(s): 82.45. $-\mathrm{h}, 02.60 .-\mathrm{x}$

\section{INTRODUCTION}

The behavior of charges in liquids under the influence of an applied voltage is a very general problem. However, even when only drift and diffusion of the charges are considered (neglecting chemical reactions between charges and at interfaces), the nonlinear character of the relevant equations results in a variety of different phenomena. The behavior of an electrolyte depends mainly on the amplitude of the applied voltage and on the amount of charges in the device. Polar solvents generally contain more charges than nonpolar solvents due to their smaller Bjerrum length. However, when a charging agent is used, such as a surfactant, high charge concentrations can also occur in nonpolar solvents [1]. Applications of polar electrolytes are electrochromic devices [2] and dye sensitized solar cells [3], whereas for nonpolar electrolytes, important applications are liquid crystal displays [4], electrophoretic ink [5], and the stabilization of soot in petrol [6].

Measurements of transient currents can reveal a lot about the number, the nature, and the behavior of the charges in a liquid $[7,8]$. Typically, at time $t=0$, a voltage step from 0 to $V$ is applied over a planar structure with thickness $d$ (Fig. 1). Between the high potential electrode at position $x=-d / 2$ and the low potential electrode at $x=d / 2$ is a solvent at temperature $T$ with relative dielectric constant $\varepsilon_{r}$. This solvent contains an equal amount of positive and negative charges $\pm q$ (C), which are identical except for their polarity. The average density of charges (including positive and negative charges) is $\bar{n}\left(\mathrm{~m}^{-3}\right)$. The charges have mobility $\mu\left(\mathrm{m}^{2} \mathrm{~V}^{-1} \mathrm{~s}^{-1}\right)$ and diffusion constant $D\left(\mathrm{~m}^{2} \mathrm{~s}^{-1}\right)$, which are related by Einstein's equation $D / \mu=k T / q$, in which $k$ is Boltzmann's constant.

In this work, the situation of a high charge content (so that the Debye length $\lambda_{D}=\sqrt{\varepsilon_{r} \varepsilon_{0} k T / q^{2} \bar{n}}$ is small compared to the thickness of the device) and relatively small voltage steps (smaller than or around $k T / q$ ) is discussed analytically and compared with numerical simulations. Under these circumstances, double layer charging is initially the dominant phenomenon, resulting in an exponentially decreasing transient current [9]. Near the double layer, the concentration of posi-

\footnotetext{
*Corresponding author; matthias.marescaux@elis.ugent.be
}

tive and negative charges becomes lower than in the bulk. As a result, positive and negative charges diffuse toward the electrodes, as previously explained by Bazant et al. [10]. This diffusion of two opposite charges is not associated with a current, but the readjustment of the double layer leads to a measurable current. This transient current is negligible during the initial double layer charging, but it becomes dominant at longer times because it decreases more slowly than an exponential decay. We derive an analytical expression for this current contribution and discuss for which parameter values and during which time interval this expression is a good approximation of the total current.

\section{THEORY}

Assuming a symmetrical electrolyte and neglecting reactions between the ions, charge transport in a one-dimensional device can be described by the laws of drift and diffusion (expressed by the Nernst-Planck equation) together with the continuity equation, which are formulated as [11]

$$
\begin{aligned}
& \frac{\partial n}{\partial t}=-\mu \frac{\partial}{\partial x}\left(\frac{\rho}{q} E\right)+D \frac{\partial^{2} n}{\partial x^{2}}, \\
& \frac{\partial \rho}{\partial t}=-\mu \frac{\partial}{\partial x}(q n E)+D \frac{\partial^{2} \rho}{\partial x^{2}} .
\end{aligned}
$$

In these equations, $n(x, t)\left(\mathrm{m}^{-3}\right)$ is the total density (the sum of the densities of positive and negative charges), and $\rho(x, t)$ $\left(\mathrm{C} \mathrm{m}^{-3}\right)$ is the charge density. The electric field $E(x, t)$ $\left(\mathrm{V} \mathrm{m}^{-1}\right)$ is related to the charge density by Gauss's law $\varepsilon_{r} \varepsilon_{0} \partial E / \partial x=\rho$, and to the applied voltage by $V=\int_{-d / 2}^{d / 2} E d x$. We assume blocking electrodes, so the flux of charges and the current density at the electrodes is zero. Initially, all charges are homogeneously distributed, so that $n=\bar{n}$ and $\rho=0$.

The partial differential equations (1) and (2) are discretized and the values for the next time step are found by using an iterative method. The backward Euler scheme is an implicit method, involving the inversion of a large matrix. The intervals have different widths with smaller intervals near the edges. Sharp spatial variations in $\rho$ and $n$ are treated with Bernoulli functions [12]. The resulting simulation program is fast and accurate enough for our purposes even if 


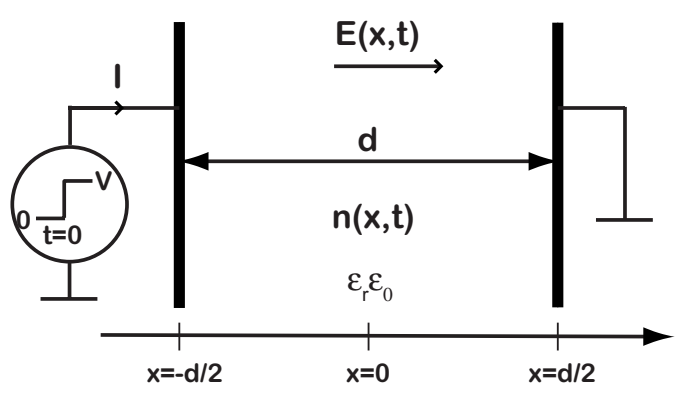

FIG. 1. Schematic representation of the model. At $t=0 \mathrm{~s}$, a voltage $V$ is applied to a homogeneous, symmetric electrolyte with relative dielectric constant $\varepsilon_{r}$ between two blocking electrodes at a distance $d$ from each other.

high charge concentrations or large voltage steps are considered.

For low voltages $(V \ll k T / q)$, the dominant role of diffusion allows one to assume that the total density $n$ is approximately constant and homogeneous. The steady-state solution $\rho_{S S}(x)\left(\mathrm{C} \mathrm{m}^{-3}\right)$ of the charge density can then be found by equating the time derivative in Eq. (2) to zero,

$$
\rho_{S S}=q \bar{n} \frac{q V}{2 k T} \frac{\sinh \left(\frac{x}{\lambda_{D}}\right)}{\sinh \left(\frac{d}{2 \lambda_{D}}\right)} \text {. }
$$

The steady-state electric field corresponding with Eq. (3) can then be found using Gauss's law,

$$
E_{S S}=\frac{V}{2 \lambda_{D}} \frac{\cosh \left(\frac{x}{\lambda_{D}}\right)}{\sinh \left(\frac{d}{2 \lambda_{D}}\right)} .
$$

Substituting these results back in Eq. (1), and assuming $\lambda_{D} \ll d$, the following solution for the total density in steady state is found:

$$
n_{S S}=\bar{n}\left[1-\frac{1}{2}\left(\frac{q V}{2 k T}\right)^{2} \frac{\lambda_{D}}{d}\right]+\bar{n} \frac{1}{2}\left(\frac{q V}{2 k T}\right)^{2} \frac{\cosh \left(\frac{2 x}{\lambda_{D}}\right)}{\sinh \left(\frac{d}{\lambda_{D}}\right)} .
$$

Notice that the characteristic screening length is different for $n_{S S}\left(\lambda_{D} / 2\right)$ in comparison with $\rho_{S S}\left(\lambda_{D}\right)$. For high charge contents $\left(\lambda_{D} \ll d\right)$, the dynamics of the charge density is well described by a single exponential [9],

$$
\rho=\rho_{S S}\left[1-\exp \left(-\frac{2 D t}{\lambda_{D} d}\right)\right] .
$$

Using Ramo's theorem $[13,14]$, one can find the external electric current $I(t)$ that corresponds to this double layer charging (with $S$ the area of the device),

$$
I_{D L}=S q \bar{n} \mu \frac{V}{d} \exp \left(-\frac{2 D t}{\lambda_{D} d}\right)
$$

which results in a charge $Q_{e l, S S}$ on the electrodes in steady state given by

$$
Q_{e l, S S}=\frac{\varepsilon_{r} \varepsilon_{0} S}{\lambda_{D}} \frac{V}{2} .
$$

The dynamics of the total density $n$ is more complicated. The concentration of charges near the electrodes, in a layer with thickness of the order $\lambda_{D} / 2$, is built up by drift and diffusion with roughly the same time constant as the double layer charge [9], $\lambda_{D} d / 2 D$. Equation (5) shows that, in steady state, this build up near the interfaces is compensated by a small decrease of the total density over the whole thickness of the device. However, this homogeneous decrease cannot occur on the short time scale of double layer charging. The charges which build up the concentration near the interfaces in a time $\lambda_{D} d / 2 D$ can therefore only have come from a region near the interfaces with a width of the order of the diffusion length on this time scale. This becomes obvious if one considers the case $\lambda_{D} \ll d$. In this case, a very thin double layer (with width $\lambda_{D}$ ) builds up in a very short time interval $\lambda_{D} d / 2 D$. These charges have arrived there by diffusion from a region near the electrodes with thickness in the order of $\sqrt{\lambda_{D} d / 2}$, which is larger than the double layer, but still very thin. These diffusion layers are subsequently filled up, over a much longer time scale, by diffusion of charges from the whole device. The evolution of the total density in these layers can be described by the Gaussian diffusion of two very thin layers [10], containing a total amount of charges opposite to the integrated total density in the double layers $\bar{n} \frac{\lambda_{D}}{2}\left(\frac{q V}{2 k T}\right)^{2}$, resulting in

$$
\begin{aligned}
n_{\text {diff }}= & -\bar{n} \frac{\lambda_{D}}{2}\left(\frac{q V}{2 k T}\right)^{2} \frac{1}{2 \sqrt{\pi D t}}\left[\exp \left(-\frac{(x+d / 2)^{2}}{4 D t}\right)\right. \\
& \left.+\exp \left(-\frac{(x-d / 2)^{2}}{4 D t}\right)\right] .
\end{aligned}
$$

It should be noted that Eq. (9) assumes a sufficiently large device thickness. As time progresses, this assumption will not remain valid, because the diffusion length becomes comparable to the thickness $d$. This also implies that Eq. (9) approaches 0 if large times (steady state) are considered which means that the conservation of the number of charges is violated. If both boundaries of the sample are taken into account, the evolution of the diffusion layers can be described by

$$
n_{\text {diff }}=-\bar{n} \frac{\lambda_{D}}{2}\left(\frac{q V}{2 k T}\right)^{2} \frac{1}{2 \sqrt{\pi D t}} \sum_{k=-\infty}^{+\infty} \exp \left(-\frac{[x+(2 k-1) d / 2]^{2}}{4 D t}\right) .
$$

At all times, $\int_{-d / 2}^{d / 2} n_{\text {diff }} d x=-\bar{n} \frac{\lambda_{D}}{2}\left(\frac{q V}{2 k T}\right)^{2}$, so the total amount of charges $\int_{-d / 2}^{d / 2} n d x=\bar{n} d$ is conserved [see Eq. (5)]. Figure 2 shows a simulation illustrating the diffusion of charges from the bulk to the surfaces. 

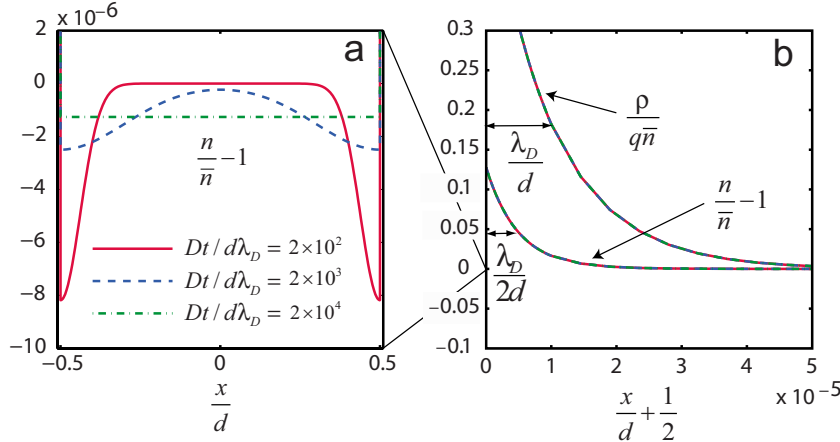

FIG. 2. (Color online) Simulations illustrating the principle of the evolution of the diffusion layers and their impact on the double layer. The spreading of the Gaussian profiles (a), describing diffusion of both charges, is shown for $D t / d \lambda_{D}=2 \times 10^{2}$ (solid red line), $2 \times 10^{3}$ (dashed blue line), and $2 \times 10^{4}$ (dashed-dotted green line) $\left(\lambda_{D} / d=10^{-5}\right.$ and $\left.q V / k T=1\right)$. The thickness and the height of the diffuse double layer for $n$ and $\rho$ (b) changes only a little for different $D t / d \lambda_{D}$ (not visible in this figure).

The evolution of the diffusion layers has an indirect effect on the charge density (and thus the current), because the diffusion layers lead to a correction on the original assumption that leads to Eqs. (3), (4), and (6)-(8). These equations were calculated assuming that the total density just outside of the double layer is equal to the average total density $\bar{n}$. Evaluating Eq. (9) at $x= \pm d / 2$ provides a correction on this value, so that the total density just outside the double layer is (for $t \ll d^{2} / D$ ) better described by

$$
\bar{n}^{\prime}=\bar{n}\left[1-\frac{\lambda_{D}}{2}\left(\frac{q V}{2 k T}\right)^{2} \frac{1}{2 \sqrt{\pi D t}}\right] .
$$

Similarly, a better approximation for the width of the double layer is given by $\lambda_{D}^{\prime}=\sqrt{\varepsilon_{r} \varepsilon_{0} k T / q^{2} \bar{n}^{\prime}}$. For times which are larger than the charging time of the double layer [see Eq. (6)] but smaller than $t \ll d^{2} / D$ (diffusion has not reached the other side of the device), the charge on the electrodes can be approximated by

$$
Q_{e l}=\frac{\varepsilon_{r} \varepsilon_{0} S}{\lambda_{D}^{\prime}} \frac{V}{2}=\frac{\varepsilon_{r} \varepsilon_{0} S}{\lambda_{D}} \frac{V}{2}\left[1-\frac{1}{2} \frac{\lambda_{D}}{2}\left(\frac{q V}{2 k T}\right)^{2} \frac{1}{2 \sqrt{\pi D t}}\right] .
$$

This charge depends on time and yields a transient current,

$$
I_{\text {diff }}=\frac{\varepsilon_{r} \varepsilon_{0} S}{\sqrt{\pi D}} \frac{V}{2}\left(\frac{q V}{8 k T}\right)^{2} t^{-3 / 2} .
$$

Equation (12) is valid for voltage steps smaller than $k T / q$. If, however, intermediate voltages $(q V / k T>1)$ are considered, Eq. (9) is no longer valid. For intermediate voltages, a different voltage dependency for the total density has been proposed in a paper by Bazant et al. [10],

$$
\begin{aligned}
n_{\text {diff }}= & -\bar{n} \frac{\lambda_{D}}{2} \sinh ^{2}\left(\frac{q V}{2 k T}\right) \frac{1}{2 \sqrt{\pi D t}}\left[\exp \left(-\frac{(x+d / 2)^{2}}{4 D t}\right)\right. \\
& \left.+\exp \left(-\frac{(x-d / 2)^{2}}{4 D t}\right)\right] .
\end{aligned}
$$

Based on Eq. (14) instead of (9), we can repeat the derivation above using the total density at $x= \pm d / 2$ for $t$ $\ll d^{2} / D$. In this way, we obtain the transient current, associated with the variation of the diffusion layers,

$$
I_{\text {diff }}=\frac{\varepsilon_{r} \varepsilon_{0} S}{\sqrt{\pi D}} \frac{V}{2} \sinh ^{2}\left(\frac{q V}{8 k T}\right) t^{-3 / 2} .
$$

For low voltages, Eq. (15) reduces to Eq. (13).

\section{DISCUSSION}

Figure 3 shows the transient currents that are obtained with the numerical simulation program described above, if the applied voltage switches from zero to four different val-

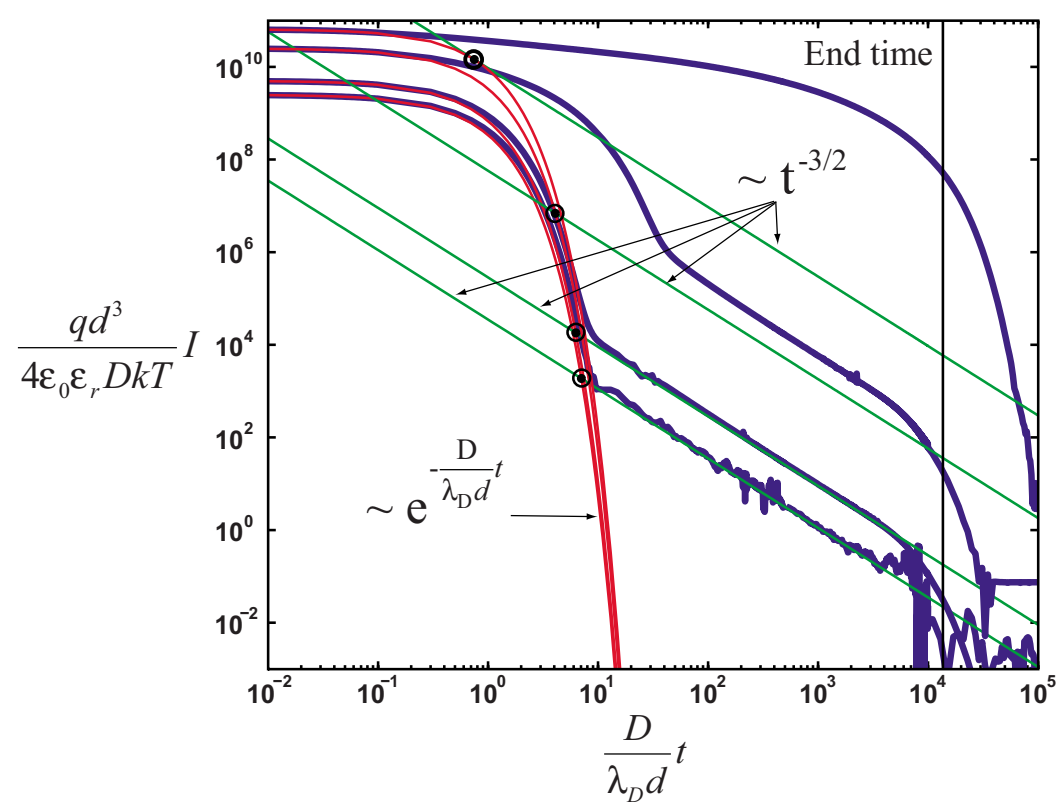

FIG. 3. (Color online) Simulations of transient currents in response to different voltage steps, $\frac{q V}{k T}=1,2,10$, and 25.918 (for which $\tau_{\text {diff }} / \tau_{D L}=3 / 2$ ) for $\lambda_{D} / d=10^{-5}$ (blue lines). The transient currents are compared with both analytical fits, the exponential [Eq. (7)] (red lines) and the power-law decay [Eq. (15)] (green lines), showing good agreement for the lowest voltages. The intersection point between the exponential and the power-law decay is drawn (dots) as well as the end of the validity of the $-3 / 2$ power-law decay for which $D t / \lambda_{D} d \ll d / 8 \lambda_{D}$ (vertical black line). 
ues. The simulation results are compared with the analytical expressions in Eqs. (7) and (15). The initial part of the transient current is best described by the exponential curve corresponding to double layer charging [Eq. (7)]. For the three lowest voltages, there is a time interval where the simulated current is well described by the formula for the spreading of diffusion layers [Eq. (15)]. The noise in the simulated transient current at larger times $\left(D t / \lambda_{D} d>10\right)$ is due to the division of two small numbers in the algorithm. For the highest voltage, the correspondence between the simulations and the analytical model gets worse because the electric field in the bulk is no longer negligible.

The currents given by Eqs. (7) and (15) intersect at a certain time $\tau_{\text {diff, }}$, as long as the applied voltage is sufficiently small. The value of $\tau_{\text {diff }}$ is found by setting the currents in both equations equal, resulting in

$$
\sinh ^{2}\left(\frac{q V}{8 k T}\right)=\sqrt{\frac{\pi d}{2 \lambda_{D}}}\left(\frac{\tau_{\text {diff }}}{\tau_{D L}}\right)^{3 / 2} \exp \left(-\frac{\tau_{\text {diff }}}{\tau_{D L}}\right),
$$

with $\tau_{D L}=\lambda_{D} d / 2 D$ the time constant for double layer charging. The right-hand side of this equation reaches a maximum at $\tau_{\text {diff }} / \tau_{D L}=3 / 2$ and the corresponding voltage $V^{*}$ can be found by substituting this value in Eq. (16); $\frac{q V^{*}}{k T}=25.918$. For voltages higher than $V^{*}$, the two analytical solutions do not intersect anymore, as can be seen in Fig. 3. These limits correspond roughly with the conditions for double layer limited current, explained in Ref. [11].

In order to find Eq. (15), we have assumed that the diffusion occurs in an infinite medium. This leads to another limitation for Eq. (15), namely, that the diffusion length $\sqrt{2 D t}$ should be smaller than $d / 2$ because both diffusion fronts of Eq. (9) start to overlap at the middle of the device. This means that Eq. (15) is only valid for times satisfying the condition $D t / \lambda_{D} d \ll d / 8 \lambda_{D}$. This value is indicated in Fig. 3 as the end time.

\section{CONCLUSION}

This work shows that double layer charging cannot be described consistently without taking the effect of diffusion of charges into the bulk of the device into account. Although this diffusion results in a small correction on the bulk total density, there is an important transient current associated with it, which is dominant on a longer time scale. The fact that there is a typical $-3 / 2$ power-law transient current, associated with the evolution of the diffusion layers, enables an indirect measurement. A full numerical calculation method is presented together with analytical approximations for the current at short and long time scales.
[1] I. D. Morrison, Colloids Surf., A 71, 1 (1993).

[2] S. K. Deb and J. A. Chopoorian, J. Appl. Phys. 37, 4818 (1966).

[3] B. O’Regan and M. Grätzel, Nature (London) 335, 7377 (1991).

[4] G. Stojmenovik, S. Vermael, K. Neyts, R. Van Asselt, and A. R. M. Verschueren, J. Appl. Phys. 96, 3601 (2004).

[5] B. Comiskey, J. D. Albert, H. Yoshizawa, and J. Jacobson, Nature (London) 394, 253 (1998).

[6] Y. Won, S. P. Meeker, V. Trappe, and D. A. Weitz, Langmuir 21, 924 (2005).

[7] F. Strubbe, A. R. M. Verschueren, L. J. M. Schlangen, F. Beunis, and K. Neyts, J. Colloid Interface Sci. 300, 396 (2006).
[8] V. Novotny, J. Electrochem. Soc. 133, 1629 (1986).

[9] F. Beunis, F. Strubbe, M. Marescaux, and K. Neyts, Appl. Phys. Lett. 91, 182911 (2007).

[10] M. Z. Bazant, K. Thornton, and A. Ajdari, Phys. Rev. E 70, 021506 (2004).

[11] F. Beunis, F. Strubbe, M. Marescaux, J. Beeckman, K. Neyts, and A. R. M. Verschueren, Phys. Rev. E 78, 011502 (2008).

[12] S. Selberherr, Analysis and Simulation of Semiconductor Devices (Springer-Verlag, Wien, New York, 1984), pp. 191-197.

[13] P. De Visschere, Solid-State Electron. 33, 455 (1990).

[14] K. Neyts, J. Beeckman, and F. Beunis, Opto-Electron. Rev. 15, 41 (2007). 